\title{
Fast ion instability at the Cornell Electron Storage Ring Test Accelerator
}

\author{
A. Chatterjee, ${ }^{*}$ K. Blaser, W. Hartung, D. Rubin, and S. T. Wang \\ Cornell Laboratory for Accelerator-based Sciences and Education, Cornell University, \\ Ithaca, New York, USA
}

(Received 12 September 2014; published 29 June 2015)

\begin{abstract}
Fast ion instability can lead to deterioration of an electron beam (increasing emittance and instability of a train of bunches) in storage rings and linacs. We study this at the Cornell electron storage ring test accelerator using a $2.1 \mathrm{GeV}$ low emittance beam. As the source of ions is residual gas, our measurements are conducted at various pressures, including nominal vacuum as well as injected gas ( $\mathrm{Ar}, \mathrm{Kr}$ ). We experiment with mitigation techniques, including changing the bunch pattern to have mini-trains instead of one long train, as well as increasing the initial vertical emittance of the beam. We also check to ensure that ion-trapping is not a substantial effect in our measurements. We measure turn-by-turn vertical bunch size and position, as well as the multibunch power spectrum. Our measurements confirm fast ion instability under all vacuum conditions. A detailed simulation is then used to compare theory with observations.
\end{abstract}

DOI: 10.1103/PhysRevSTAB.18.064402

PACS numbers: 29.27.-a, 29.20.db

\section{INTRODUCTION}

The residual gas in the accelerator vacuum system can be ionized by a circulating electron beam. The positively charged ions are trapped in the potential well of the electron bunch train. The transverse motion of the lead bunch in the train will be transferred to the ions and then from the ions to the next bunch in the train. In this way, the motion of the bunches near the head of the train is coupled to the bunches near the tail. The resulting instability limits the total charge in each bunch and the number of bunches in the train. It is observed in linear accelerators as well as electron storage rings. In a storage ring, having a long charge-free gap at the end of the train prevents multiturn ion trapping. However, this cannot prevent ions from accumulating during a single passage of the bunch train, a phenomenon referred to as fast ion instability (FII) [1].

In 2008, the Cornell electron storage ring (CESR) was reconfigured as a test accelerator (CESR-TA) to study electron and positron beam dynamics [2]. Such studies provide insight into phenomena that are likely to limit the performance of next-generation colliders and storage rings (e.g., intra-beam scattering, electron cloud growth, and FII). FII has been qualitatively observed at many accelerator facilities: by injecting gas or turning off vacuum pumps [3-5], or by reducing the beam emittance to increase the trapping potential under nominal vacuum [6]. However, the instrumentation available at CESR-TA makes it possible to measure effects of FII on individual bunches rather

*ac448@cornell.edu

Published by the American Physical Society under the terms of the Creative Commons Attribution 3.0 License. Further distribution of this work must maintain attribution to the author(s) and the published article's title, journal citation, and DOI. than the beam as a whole. The CESR beam position monitor (CBPM) system is capable of measuring bunchby-bunch, turn-by-turn horizontal and vertical beam motion with a resolution of about $10 \mu \mathrm{m}$ [7]. The X-ray beam size monitor (xBSM) is able to measure bunch-by-bunch, turnby-turn vertical beam size at the $10-100 \mu \mathrm{m}$ range [8].

We first reported on FII measurements at CESR-TA based on data collected in December 2013 [9]. In this paper, we present an update with data from the April 2014 run. The main focus here, discussed in depth in Sec. II, is the measurements done at various pressures of injected gas, as we were able to establish higher pressures than we explored previously. Section III explains the simulation software implemented at CESR-TA that is used to make direct comparisons with measurements. In Sec. IV, we present results that establish that ion-trapping is not significant in our measurements. We also probe how FII is mitigated when a single, long train is split into mini-trains, and test the effectiveness of increasing the nominal vertical emittance as a mitigation strategy. In Sec. V, we take a brief look at measurements that are not presently well understood, and will form the basis for future research. We present our conclusions in Sec. VI.

\section{MEASUREMENTS}

The measurements were done over several shifts. In general, we do not compare data taken during different shifts with each other, because machine operation conditions cannot be reproduced exactly. The nominal vacuum pressure in CESR is $1 \mathrm{nTorr}$. We were able to establish three pressures of $\mathrm{Kr}(\sim 10,17$ and $25 \mathrm{nTorr})$ during one shift, and four pressures of $\operatorname{Ar}(\sim 10,15,20$, and 25 nTorr $)$ during another. These pressure "bumps" occupied about a $10 \mathrm{~m}$ portion of the CESR ring (circumference $\sim 768 \mathrm{~m}$ ). Established pressures are uniform to $10-20 \%$. The pressures mentioned are 

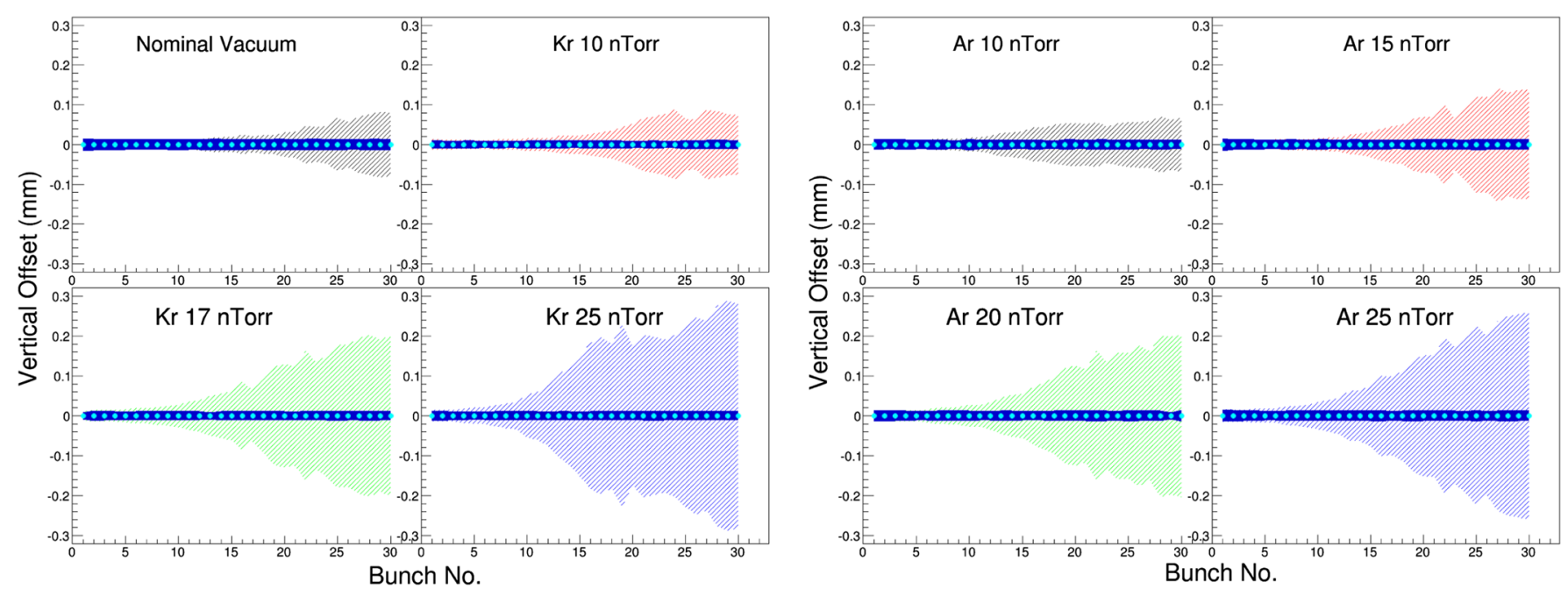

FIG. 1. Vertical bunch motion as a function of bunch number at different pressures (lightly shaded $=$ RMS amplitude with vertical feedback off, filled $=$ feedback on, dots $=$ mean value).

measured gauge pressures, and are related to the true pressure by a simple correction factor. The measurements in this section are with a 30 bunch train with $0.75 \mathrm{~mA} /$ bunch $\left(1.2 \times 10^{10}\right.$ particles $)$ and $14 \mathrm{~ns}$ bunch spacing. For each pressure, we measured $4 \mathrm{k}$ turns of CBPM data, $1 \mathrm{k}$ turns of xBSM data, and the power spectrum of the train. Each of these measurements was done with (as well as without) multibunch vertical feedback so we could determine if there is incoherent emittance growth due to the ions.

\section{A. Beam position monitor (BPM) data}

Figure 1 shows the vertical motion of each bunch (the band depicts the RMS amplitude) as a function of bunch number, with bunch 1 denoting the head of the train. The lightly shaded regions show the motion when the vertical feedback system is turned off, and the filled regions show the motion with the feedback turned on. As the pressure of the injected gas is increased, the amplitude of the motion becomes larger for the tail of the train, and the train is less stable. When the feedback is turned on, the motion is damped to a small RMS amplitude that is independent of pressure.

Besides looking at the RMS amplitude of bunch motion, we can also extract the position spectrum (e.g., betatron motion, synchrotron motion, etc.). The FFT of the motion of individual bunches gives the beam response at the normal mode tunes characteristic of the lattice. Figure 2 shows the amplitude at the vertical betatron tune for each bunch along the train from head to tail. We observe that as the pressure is increased, the growth in the amplitude along the train becomes stronger, consistent with our expectations, since the ion density increases linearly with gas pressure [1].

The vertical displacements of the bunches along the train in a single passage can be characterized by an amplitude and wavelength that are determined by the ion oscillation frequencies. We can then infer the oscillation pattern of the ions from a measurement of the relative phase of the bunch positions along the train at fixed time, as described in [10]. To do this, we create a rectangular matrix of dimension $T \times B$, where $T$ represents the number of turns of BPM data, and $B$ the number of bunches. This matrix is populated by the vertical position of the $B$ bunches over $T$ turns at a fixed BPM location. This is called the position history matrix, and is denoted by $\mathbf{P}$. The method of singular value decomposition (SVD) allows us to write $\mathbf{P}$ as a product of three matrices [11]:

$$
\mathbf{P}=\mathbf{T} \Lambda \Pi^{T}=\sum_{i=1}^{B} \tau_{i} \lambda_{i} \pi_{i}
$$
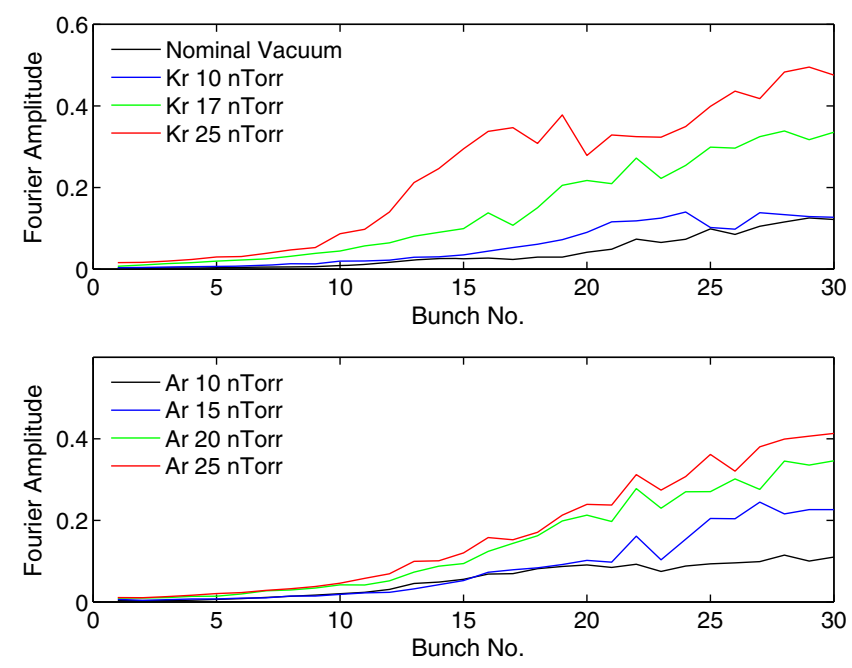

FIG. 2. Amplitude at vertical tune, obtained from FFT of vertical bunch motion (in $\mathrm{mm}$ ), as a function of bunch number at different pressures. 

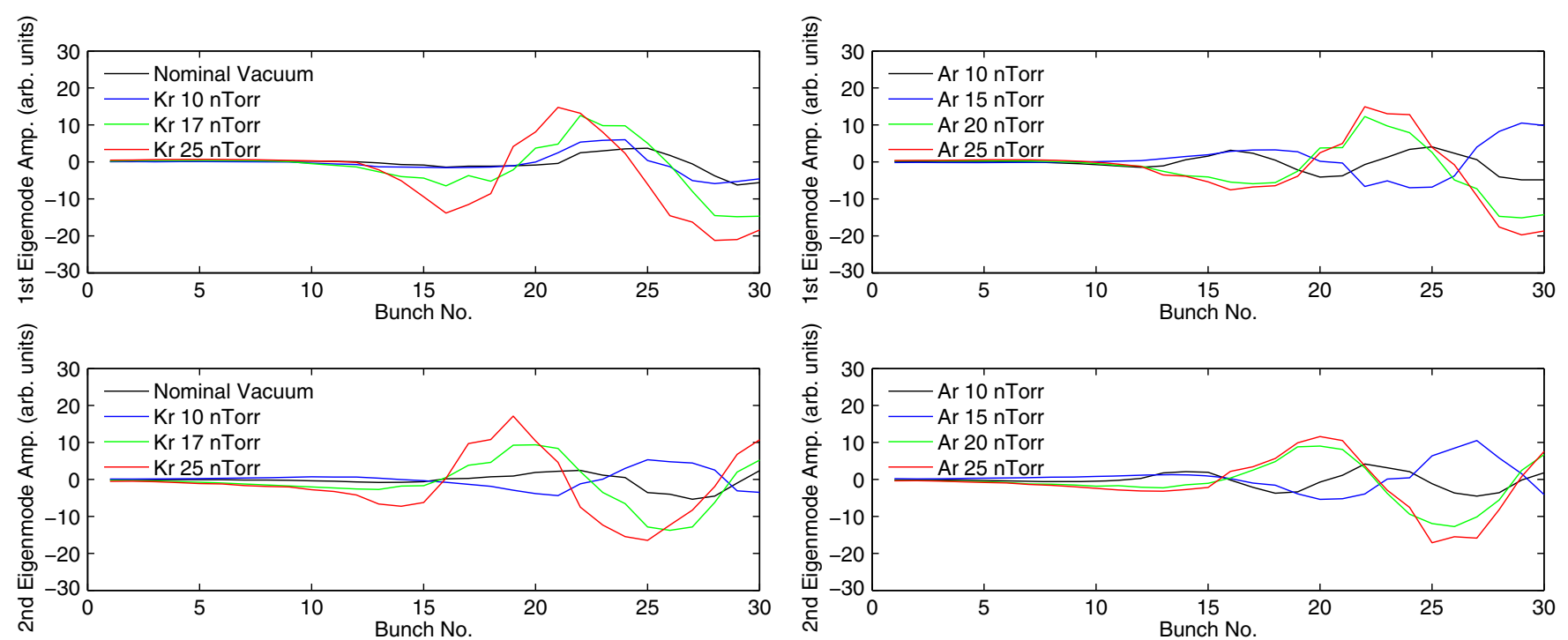

FIG. 3. Top two spatial eigenmodes (spatial eigenmodes being a representation of ion oscillations) at different pressures.

T denotes the temporal matrix, and is composed of $B$ vectors, each of length $T$. $\Pi$ denotes the spatial matrix, and is composed of $B$ vectors, each of length $B$. The temporal and spatial matrices are orthonormal. The rectangular matrix, $\Lambda$, of size $T \times B$, is a diagonal matrix. SVD is also referred to as eigendecomposition; the eigenvalues are denoted by $\lambda_{i} ; \tau_{i}$ and $\pi_{i}$ are the temporal and spacial eigenvectors, respectively. We can order the eigenvectors based on descending eigenvalues, and the top eigenvectors then correspond to the most important eigenmodes. The $\tau$ eigenvectors correspond to beam oscillation, whereas the $\pi$ eigenvectors correspond to ion oscillation.

We can multiply the $\pi$ eigenvectors with the corresponding eigenvalues to get ion oscillation modes on a fixed (albeit arbitrary) scale; these modes can now be compared. Figure 3 shows the top two spatial eigenmodes at different

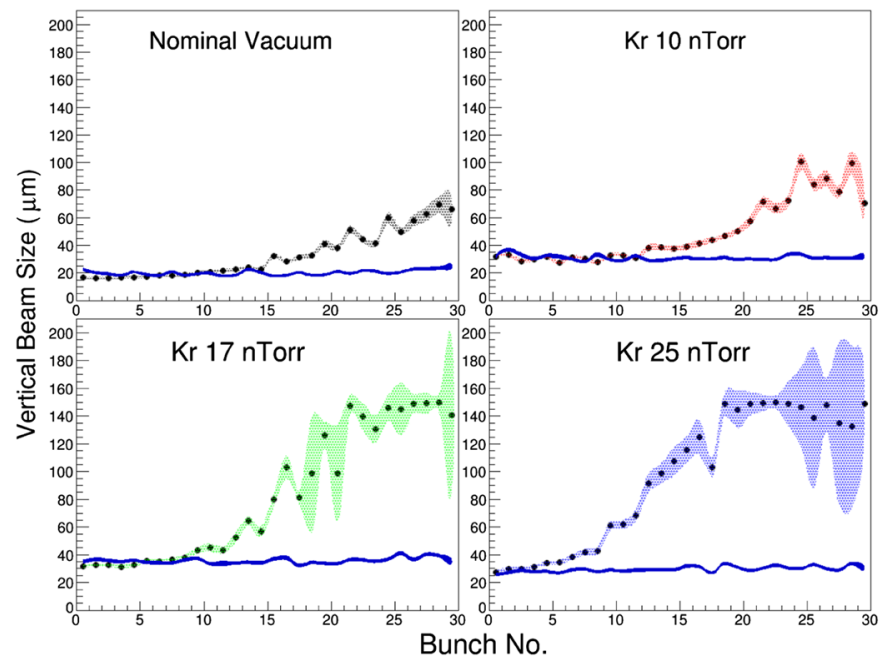

pressures of injected gas. As the pressure of the injected gas is increased, the amplitudes of the eigenmodes increase. In Sec. III, we will see that this observed pressure dependence is confirmed by simulation.

\section{B. XBSM data}

Figure 4 shows the vertical beam size of each bunch as measured by the xBSM (the band depicts the RMS uncertainty of the measurement) as a function of bunch number. The lightly shaded regions show the beam size when the vertical feedback system is turned off, and the filled regions show the beam size with the feedback on. As the pressure of the injected gas is increased, the beam size growth becomes stronger, and the measurements for the latter half of the train become more uncertain (due to

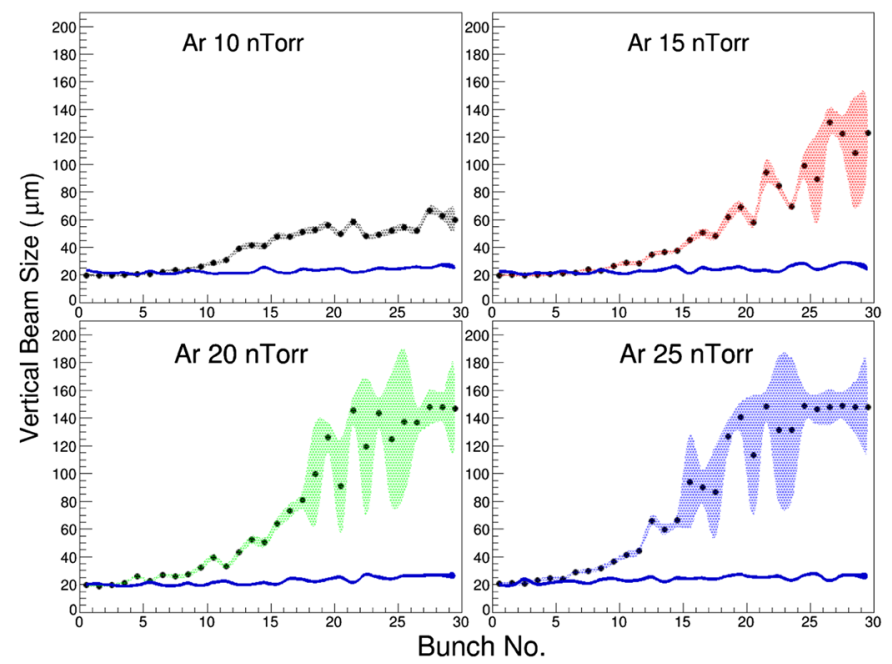

FIG. 4. Vertical bunch size as a function of bunch number at different pressures (lightly shaded $=$ RMS amplitude with vertical feedback off, filled $=$ feedback on, dots $=$ mean value) . 

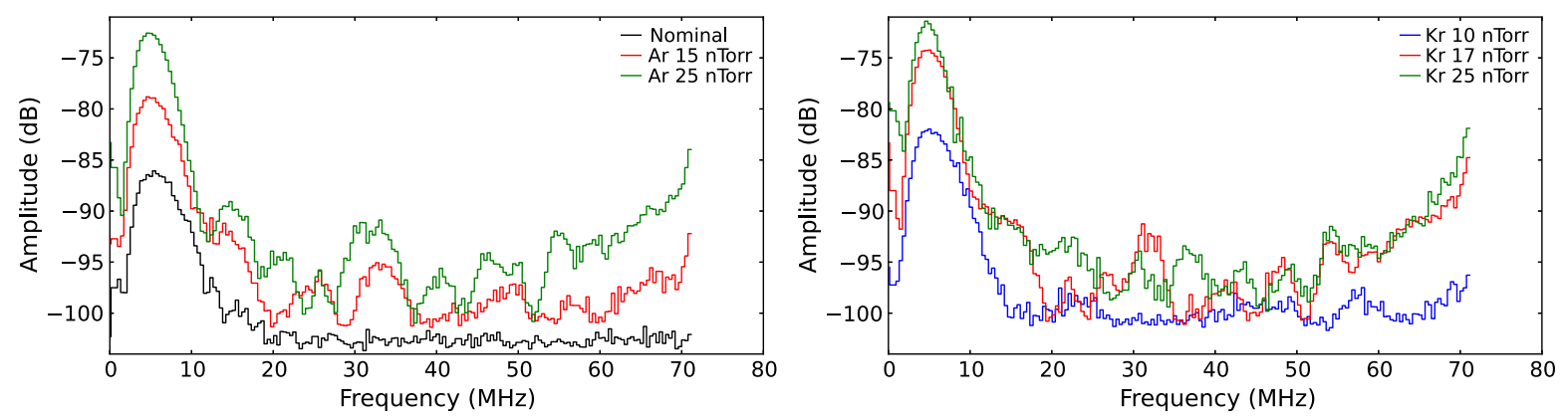

FIG. 5. Vertical lower sideband spectra at different pressures.

large beam motion as well as large beam size oscillations). The dynamic range of the XBSM is $10-150 \mu \mathrm{m}$. As a result, we see that in the higher pressure conditions, the bunch sizes for the tail of the train begin to saturate. Additionally, for the bunch size fit to be attempted, there is a cutoff on the minimum amount of incident photon flux. Hence, for later bunches in the train, where there is a lot of beam motion and thus many turns fail the requirement, the RMS uncertainty is based on a small fraction of $1 \mathrm{k}$ turns. The RMS uncertainty is thus a lower limit on the true uncertainty. When the feedback is turned on, the beam size is reduced to $\sim 20 \mu \mathrm{m}$ regardless of pressure. For the $\mathrm{Kr}$ data, the bunch size with feedback on is slightly higher, because on the shift during which the Kr data was collected, we were unable to establish the nominal machine operation conditions achieved during other shifts. We observe no incoherent growth of the vertical emittance due to the beam-ion interaction.

\section{Train spectrum data}

In addition to the bunch-by-bunch measurements described above, we record the power spectrum of the train as a whole using the signal from a single BPM as input. The bunch spacing of $14 \mathrm{~ns}$ corresponds to a frequency range of $72 \mathrm{MHz}$. Figure 5 shows the vertical lower sidebands off the revolution harmonics. The spectra for two pressures of $\mathrm{Ar}$ (viz. 10 nTorr and 20 nTorr) are missing because of problems encountered during data acquisition. We observe that as the pressure increases, so does the amplitude of the vertical lower sidebands. Since the only known multibunch instability that is affected by increasing vacuum pressure is FII, we can infer that the observed sidebands are a consequence of beam-ion coupling.

\section{SIMULATION}

We have adapted the FASTION simulation code [10], developed at CERN to study FII at the CLIC linac, to create a detailed simulation of the effects we anticipate at CESRTA. The simulation tracks electron macroparticles along the ring, creating ion macroparticles at fixed points in the ring where the beam and residual gas atoms interact with each other. Our update to the code allows the use of $6 \times 6$ matrices for beam tracking in order to include synchrotron motion. It also allows for the pressure to vary along the ring, so as to mirror our experiment (where we only increase the pressure in a small portion of the ring). We also added a rf kick, chromatic damping, and radiation damping. Finally, we include the capability to apply vertical feedback.

The simulation plots correspond to the beam behavior for the last $1 \mathrm{k}$ turns of a $25 \mathrm{k}$ turn simulation (we do not track for more turns in the interest of computation time). Since the damping time of the CESR ring is larger (about 50k turns), the beam has not reached equilibrium. However, the $25 \mathrm{k}$ turn simulation is sufficient to see whether the predicted dependence on pressure agrees with our observations. The simulations, based on a simplified model of the storage ring and the beam dynamics, necessarily provide only qualitative comparison with the measurements.

Figure 6 shows sample simulation results for nominal vacuum (defined as 0.5 nTorr each of $\mathrm{Ar}$ and $\mathrm{CO}$ ) and $25 \mathrm{nTorr}$ Ar injected at a single beam-ion interaction point.

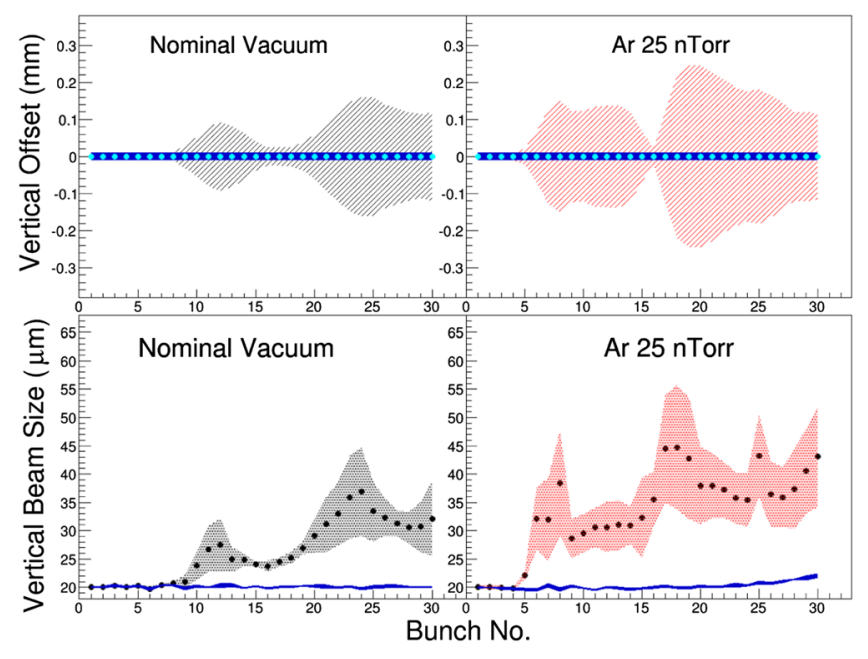

FIG. 6. Sample simulation results (14 ns spaced train with $0.75 \mathrm{~mA} /$ bunch). Vertical bunch motion (top) and size (bottom) as a function of bunch number at different pressures. (Lightly shaded $=$ RMS amplitude with vertical feedback off, filled = feedback on, dots $=$ mean value.) 


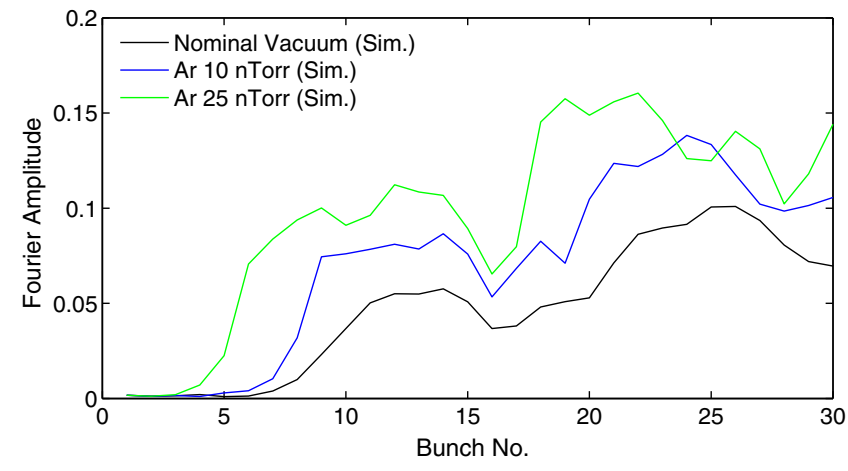

FIG. 7. Amplitude at vertical tune, obtained from FFT of vertical bunch motion (in $\mathrm{mm}$ ), as a function of bunch number at different pressures, as predicted by simulation.

The location and extent of the pressure bump used in simulation is consistent with our experiment. The ionization cross section of $\mathrm{Ar}$ and $\mathrm{CO}$ are assumed to be 1.5 and 2 MBarn [10]. Assuming a larger cross section would create a greater number of ions, increasing the instability. We see that the beam is less stable with injected gas, and that the RMS amplitude of beam motion and the growth in beam size are similar to measurements. When the feedback is turned on, the motion is damped to a small RMS amplitude that is independent of pressure, consistent with measurements.

Figure 7 shows that the amplitude of the vertical betatron motion grows along the train from head to tail. Similar to what was observed in our measurements, we notice that as the pressure is increased, the growth in the amplitude along the train becomes stronger. Moreover, for the later half of the train, the Fourier amplitude is of a similar magnitude

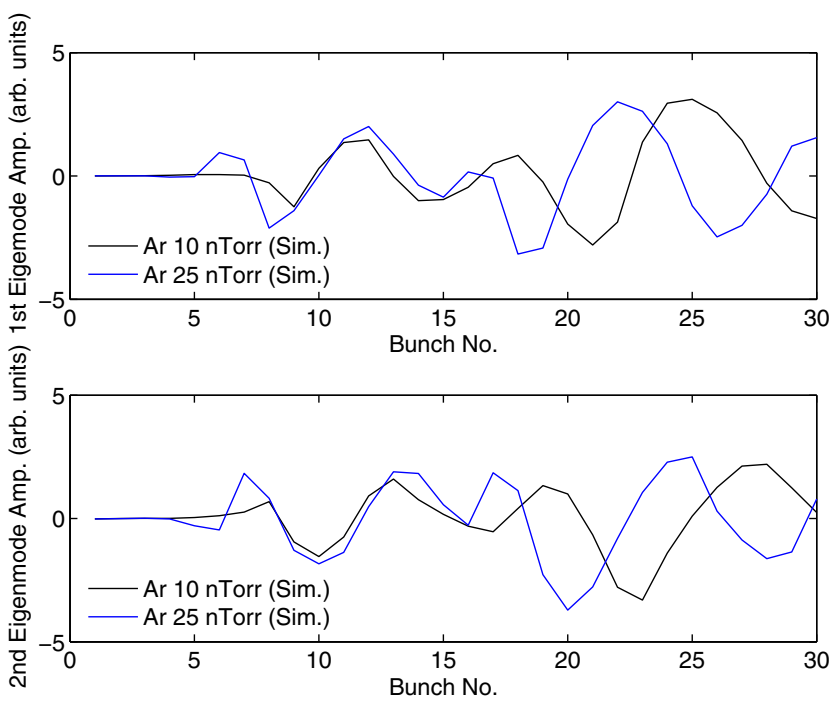

FIG. 8. Top two spatial eigenmodes (spatial eigenmodes being a representation of ion oscillations) at different pressures, as predicted by simulation. to what is measured. For the early half of the train, it is not possible for the Fourier amplitude to agree with what is measured, since the simulated bunch motion for the early bunches is too small $(<10 \mu \mathrm{m})$ compared to what the BPM can resolve. Figure 8 shows the top two spatial eigenmodes at different pressures. As the pressure of the injected gas is increased, the amplitude of the eigenmodes increases (noticeably for the early part of the train), just as was observed in our measurements. The simulated oscillation frequency is larger than what is observed. The likely reason is that the simulated beam size growth is smaller than observed, and since the oscillation frequency is inversely proportional to $\sqrt{\sigma_{y} \times\left(\sigma_{x}+\sigma_{y}\right)}\left(\sigma_{x}\right.$ and $\sigma_{y}$ denote the horizontal and vertical beam size, respectively) [1], the frequency must be higher in simulation.

\section{MITIGATION METHODS}

In this section, we begin by presenting results that establish that multiturn ion-trapping is not significant in our measurements, and that the observed instabilities are due to ions produced in a single passage of the bunch. We then probe two possible mitigation methods: splitting a single long train into smaller trains, and increasing the initial vertical emittance of the beam (to reduce the beam's ion-trapping potential [1]). The experiments described in this section were all done during a single shift with 25 nTorr (gauge pressure) of injected Ar.

\section{A. Trapping check}

We make measurements with the standard 30 bunch train, as well as a 20 bunch train. Substantial differences for the first 20 bunches for the two cases would indicate multiturn ion trapping. Figure 9a shows the vertical motion of each bunch (the band depicts the RMS amplitude) as a function of bunch number. For the short train, the motion of the first 20 bunches has slightly smaller amplitude than in the long train case, but the difference is of the same order as the reproducibility of measurements under nominally identical conditions. The difference is therefore not significant. Figure $9 \mathrm{~b}$ shows the vertical beam size of each bunch (the band depicts the RMS uncertainty of the measurement) as a function of bunch number. For the short train, the first 20 bunches have vertical size that is consistent (within $\sim 1 \sigma$ ) with the size in the long train case. Moreover, for the first ten bunches, where the measurements are most reliable, the sizes are virtually identical for the two train lengths.

Figure 10 shows the amplitude of the vertical betatron motion for each bunch. For the short train, the first 20 bunches have slightly smaller amplitude than in the long train case, but again, the differences are comparable to the reproducibility of the results. We conclude that the contribution to the observed instabilities due to multiturn ion-trapping is negligible. 


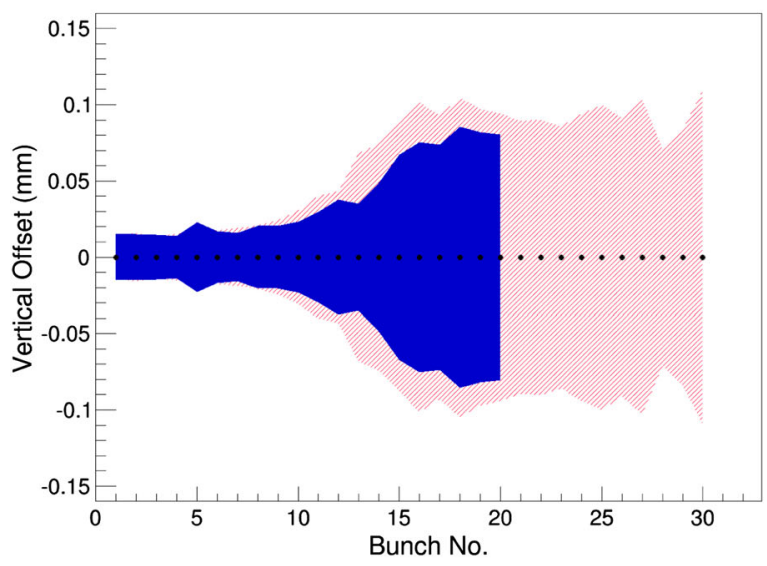

(a)

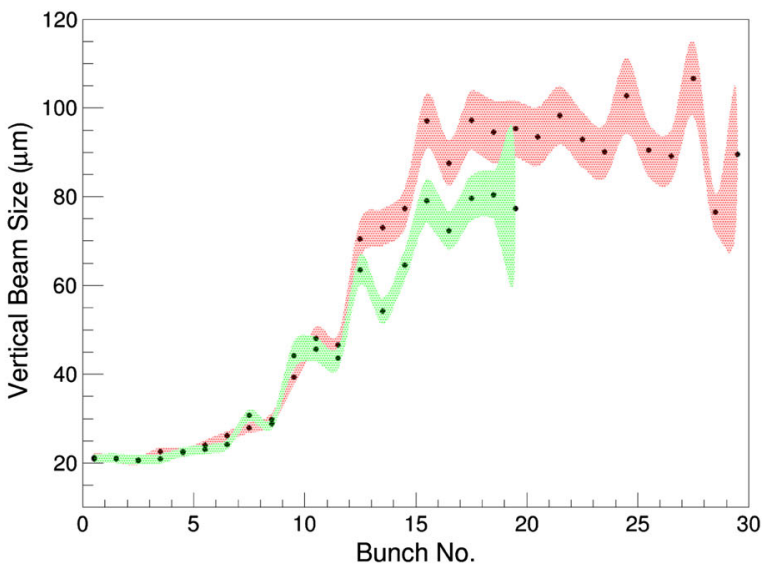

(b)

FIG. 9. Vertical bunch (a) motion and (b) size as a function of bunch number for two train lengths. (Shaded = RMS amplitude with vertical feedback off, dots = mean value.)

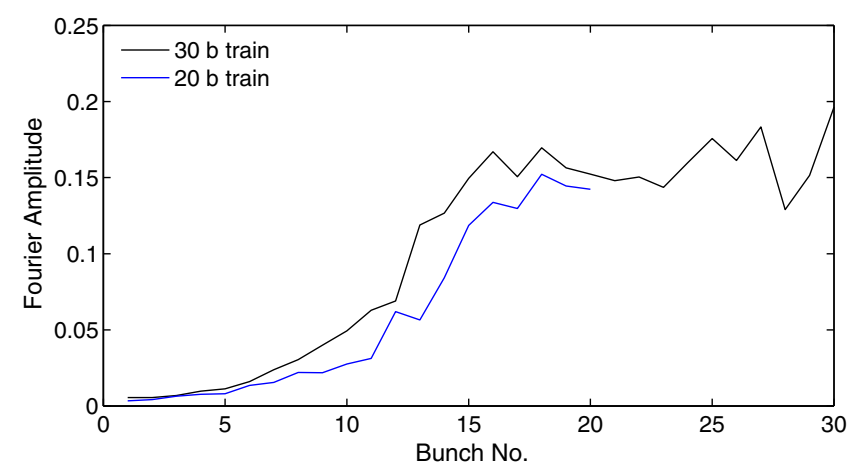

FIG. 10. Amplitude at vertical tune, obtained from FFT of vertical bunch motion (in $\mathrm{mm}$ ), as a function of bunch number for two train lengths.

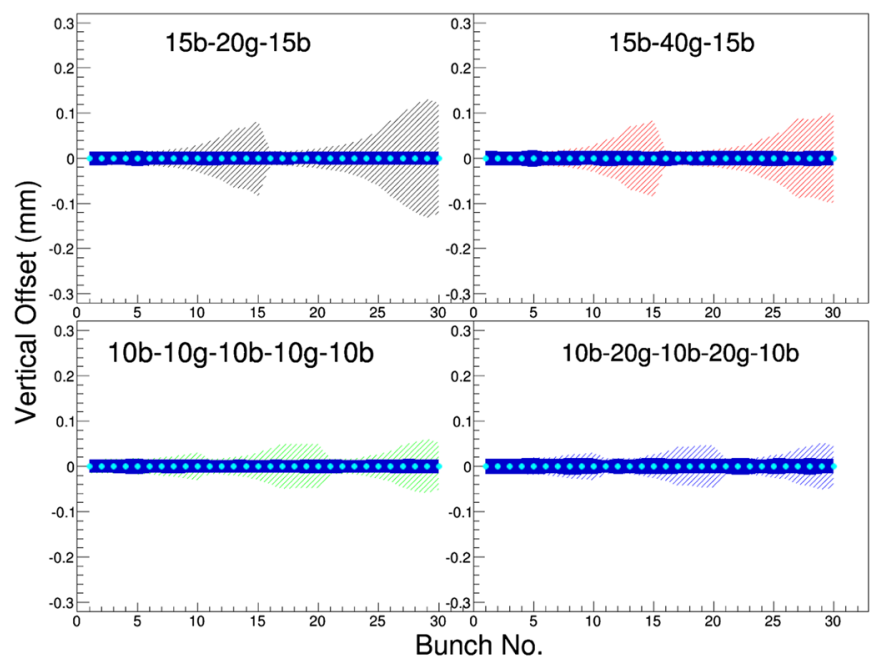

(a)

\section{B. Mini-trains}

A mitigation method that has been tried in experiment [12] and simulation [13] is to split a long train into smaller trains. We try four bunch patterns: the first two patterns have 2 mini-trains of 15 bunches each, and the next two patterns have 3 mini-trains of 10 bunches each. We denote these as "15b-20g-15b", "15b-40g-15b", "10b-10g-10b-10g-10b", and "10b-20g-10b-20g-10b", where "b" refers to a bunch, and "g" refers to a gap. Henceforth we will refer to them as patterns $1-4$, respectively. Patterns 1 and 3 occupy the same fraction of the ring (50/183), and patterns 2 and 4 occupy the same fraction of the ring (70/183); 183 is the number of available spaces when using $14 \mathrm{~ns}$ bunch spacing.

Figure 11a shows the vertical motion of each bunch (the band depicts the RMS amplitude) as a function of bunch

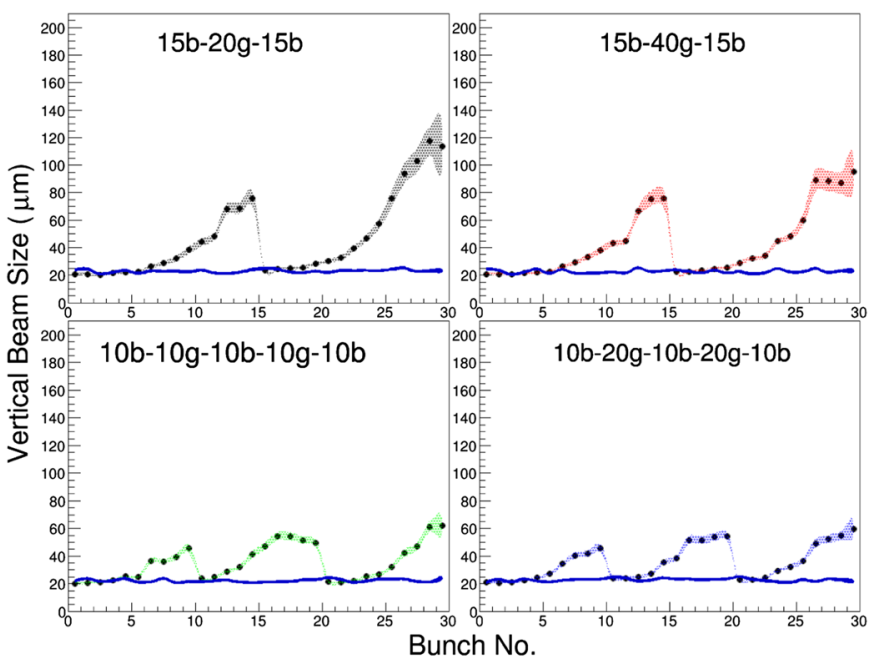

(b)

FIG. 11. Vertical bunch (a) motion and (b) size as a function of bunch number for different bunch patterns (lightly shaded = RMS amplitude with vertical feedback off, filled = feedback on, dots = mean value). In the notation used in the legends, "b" refers to a bunch, and "g" refers to a gap. 

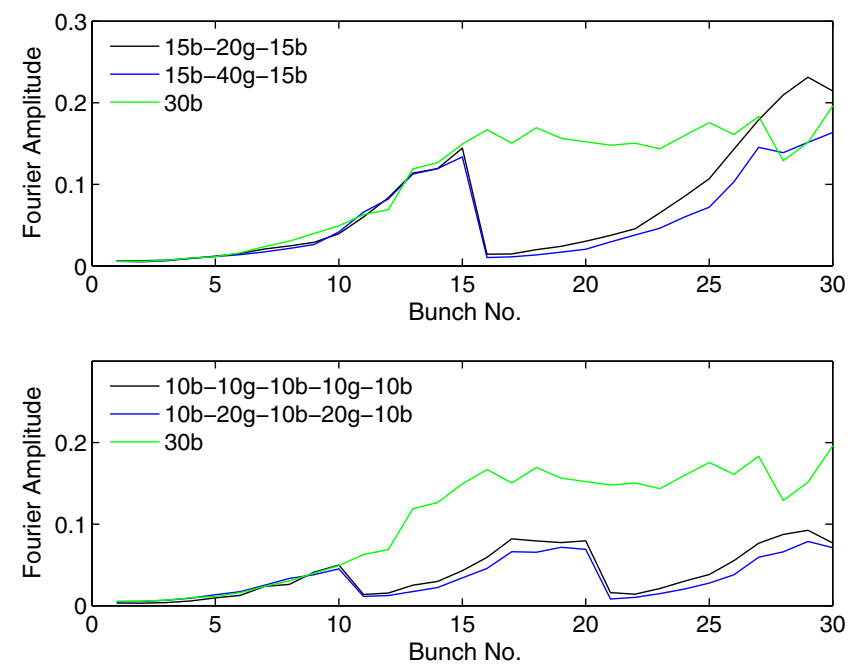

FIG. 12. Amplitude at vertical tune, obtained from FFT of vertical bunch motion (in $\mathrm{mm}$ ), as a function of bunch number for different bunch patterns. In the notation used in the legends, "b" refers to a bunch, and "g" refers to a gap.

number. We see that the second train in pattern 2 has less motion than the second train in pattern 1 , which is consistent with our expectation since the longer gap in pattern 2 allows more time for the ions to disperse. However, there is not much difference between patterns 3 and 4, because despite the longer gaps in pattern 4, the ion effects in pattern 3 are already quite weak compared to pattern 1, so the configuration is insensitive to further mitigation. By comparing patterns 1 and 3 (or patterns 2 and 4), we do see that three mini-trains are more stable than two mini-trains, consistent with what has been observed elsewhere [12]. Figure $11 \mathrm{~b}$ shows the vertical beam size of each bunch (the band depicts the RMS uncertainty of the measurement) as a function of bunch number. Keeping in line with what we observed for the BPM data, we see that (i) the second train in pattern 2 has less beam size growth than the second train in pattern 1, (ii) there is not much difference between patterns 3 and 4, and (iii) three minitrains are more stable than two mini-trains.

Figure 12 shows the amplitude at the vertical betatron tune for each bunch. We see that patterns 1 and 2 are about the same as each other (while pattern 2 is slightly lower, the difference is of a scale often seen during two sets of backto-back measurements of the same quantity), and patterns 3 and 4 are about the same as each other (with pattern 4 being slightly lower, but not in a significant way). We also note that with two mini-trains, while the amplitude is lower for bunches 16-25, by the end of the second train, the instability seems comparable to the single train case. However, with three mini-trains, the amplitude is consistently lower starting from bunch 11, i.e., three mini-trains are more stable than two mini-trains.

\section{Increased vertical emittance}

Another possible way of reducing FII would be to increase the initial vertical emittance. The vertical iontrapping potential (electric field) of the beam is inversely proportional to $\sigma_{y} \times\left(\sigma_{x}+\sigma_{y}\right)$ [12], where $\sigma_{x}$ and $\sigma_{y}$ denote the horizontal and vertical beam size, respectively. Hence, increasing the vertical emittance reduces the iontrapping potential. While of limited practical value, since we know that it is possible to introduce FII by reducing vertical emittance [6], it does provide a further test of our

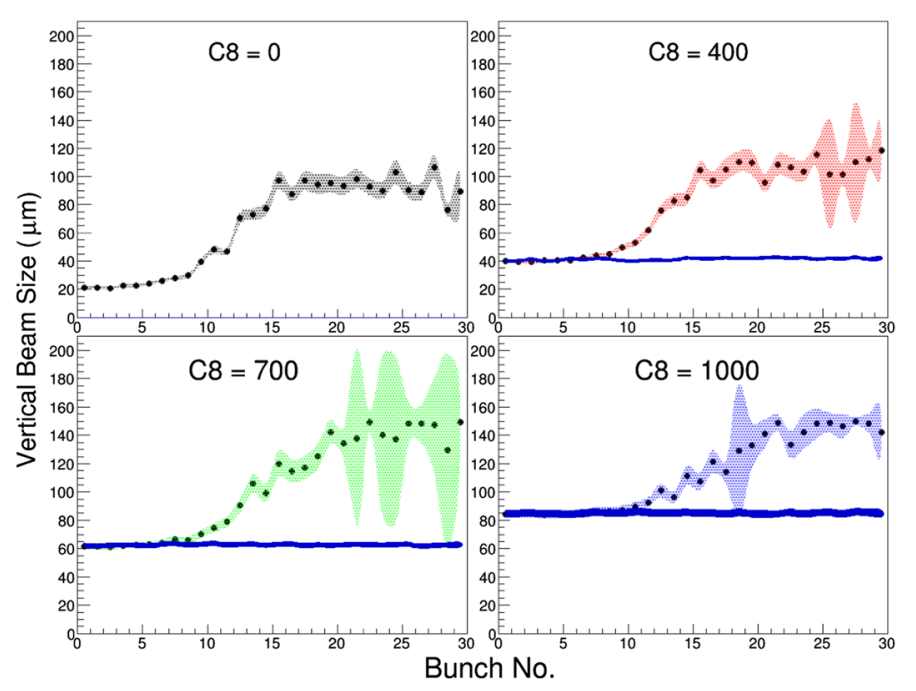

(a)

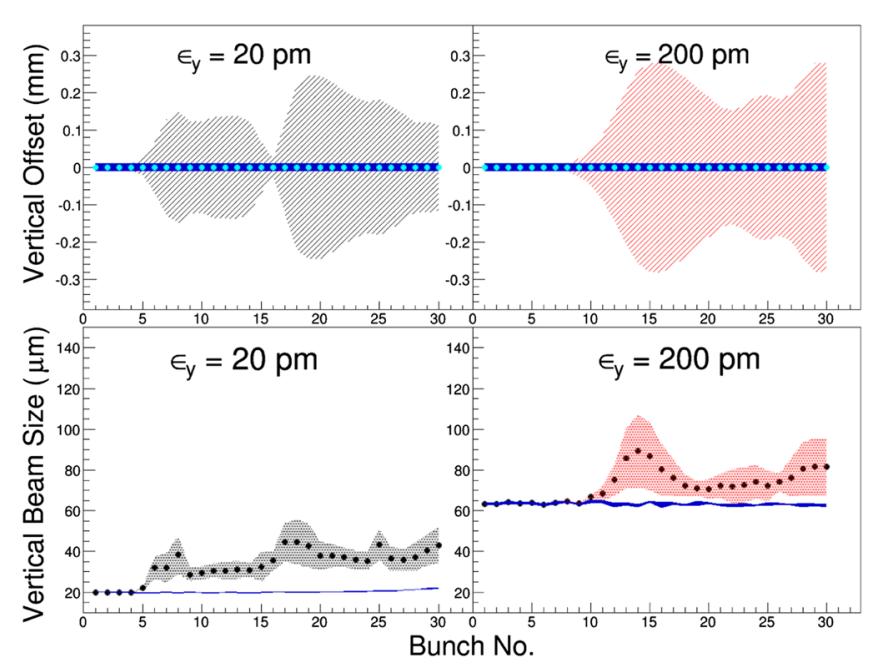

(b)

FIG. 13. (a) Vertical bunch size as a function of bunch number for different vertical emittances. (b) Simulated vertical bunch motion (top) and size (bottom) as a function of bunch number for different vertical emittances. (Lightly shaded = RMS amplitude with vertical feedback off, filled $=$ feedback on, dots $=$ mean value.) 
understanding of the fast ion instability. We were able to establish four values of initial vertical emittance (including the nominal one) by changing skew quadrupole strengths to adjust a closed coupling and vertical dispersion bump that propagates vertical dispersion through the wigglers (a tuneable parameter referenced in Fig. 13a as C8). Figure 13a shows the vertical beam size of each bunch (the band depicts the RMS uncertainty of the measurement) as a function of bunch number. As the emittance is increased, the bunch position at which beam size starts to grow is pushed back very little, whereas the maximum bunch size actually grows. Since the size of the first bunch increases from $\sim 20 \mu \mathrm{m}$ to $\sim 85 \mu \mathrm{m}$ as $\mathrm{C} 8$ is changed from 0 to 1000 , this corresponds to increasing the emittance by a factor of $(85 / 20)^{2} \approx 18$.

To understand why increasing the vertical emittance does not mitigate FII, we turn to simulation. Figure 13b corresponds to the beam behavior for the last $1 \mathrm{k}$ turns of a $25 \mathrm{k}$ turn simulation, with $25 \mathrm{nTorr}$ Ar injected at a single beam-ion interaction point. The nominal emittance used in simulation is $20 \mathrm{pm}$, comparable to what was achieved during our measurements. We see that even when the emittance is increased by an order of magnitude, the beam motion for the second half of the train still grows, and this corresponds to an increased beam size in the second half of the train. One feature seen in the simulated beam size that is mirrored in data is that the maximum bunch size seen at the higher emittance is larger than the maximum bunch size seen at the nominal emittance. There is a small discrepancy with respect to where in the train the beam size growth starts: in data it is pushed back by 3-4 bunches, whereas in simulation, this is about 5-6 bunches. Since we believe that the beam size increase is correlated with the increase in the amplitude of centroid motion, and that correlation is due at least in part to sextupole nonlinearities (not modeled in simulation), this discrepancy is not unexpected. Regardless, we conclude that when the pressure is high, increasing the initial vertical emittance is no longer an effective mitigation technique, since the decreased ion-trapping potential is not enough to compensate for the high density of ions.

\section{FUTURE WORK}

The most significant unresolved issue that emerges from our measurements is the observed current dependence of the instability threshold. All the results we have discussed thus far are for $0.75 \mathrm{~mA} /$ bunch. Figure 14 is an analog of Fig. 2; the difference is that the current is $0.5 \mathrm{~mA} /$ bunch instead of $0.75 \mathrm{~mA}$. Unlike the $0.75 \mathrm{~mA}$ case, the growth in the amplitude along the train does not increase monotonically with gas pressure. Based on theory and simulation, we have no reason to expect that $0.5 \mathrm{~mA}$ should behave differently from $0.75 \mathrm{~mA}$ in terms of FII, which suggests that there is another collective effect that is significant at $0.5 \mathrm{~mA}$, but not at $0.75 \mathrm{~mA}$. To understand this anomaly, we have further explored current and chromaticity
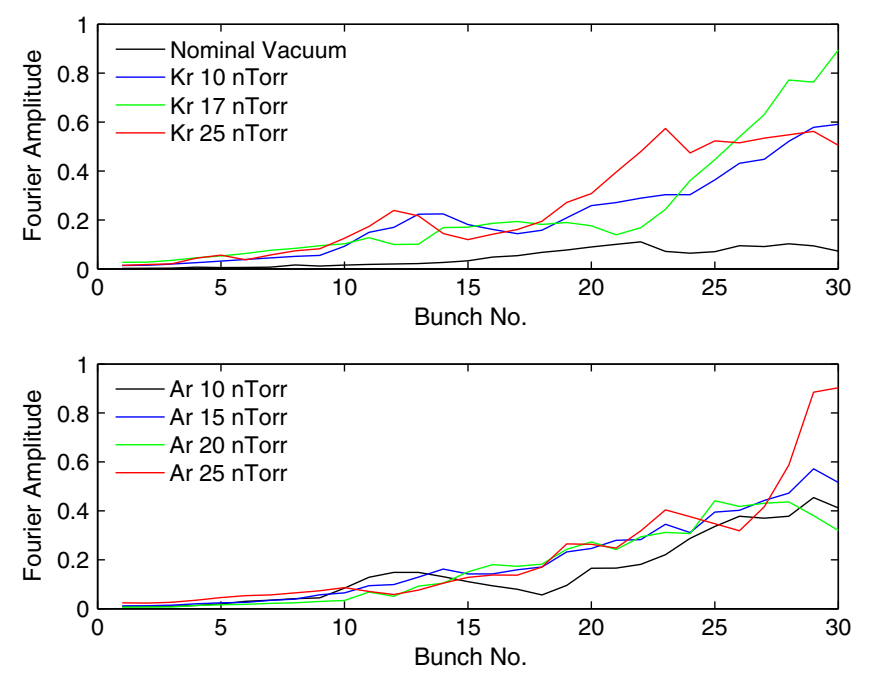

FIG. 14. Amplitude at vertical tune, obtained from FFT of vertical bunch motion (in $\mathrm{mm}$ ), as a function of bunch number at different pressures for $0.5 \mathrm{~mA} /$ bunch current.

dependence of the instability. While this has yielded some interesting insights, we have yet to grasp the full picture, and further measurements are needed to resolve the issue.

\section{CONCLUSION}

We reported here the latest measurements of FII at CESR-TA. The observed increase in beam motion and beam size along the train is correlated with pressure, and the location (along the train) and magnitude of the instability agree well with simulation. With vertical feedback, the emittance growth due to FII is eliminated, in both measurement and simulation. We demonstrated that iontrapping is not significant for a train that occupies one-sixth of the ring's circumference. We also established the effectiveness of using mini-trains as an FII mitigation technique, and determined that increasing the nominal emittance by more than an order of magnitude is not an effective mitigation strategy when injected gas pressure is high. Current dependence of multibunch instability is not well understood, and will be the subject of future research.

\section{ACKNOWLEDGMENTS}

The experiments reported here would not have been possible without the diligent support of the CESR Operations Group, the CESR-TA XBSM group (particularly Brian Heltsley), and Yulin Li who established and monitored the various vacuum pressures. A special thank you to Kiran Sonnad for his help in implementing the simulation software. This work is supported by NSF and DOE Contracts No. PHY-0734867, No. PHY-1002467, No. PHYS-1068662, No. DE-FC02-08ER41538, No. DESC0006505, and the Japan/U.S. Cooperation Program. 
[1] T. Raubenheimer and F. Zimmermann, Phys. Rev. E 52, 5487 (1995).

[2] M. Palmer et al., LEPP Report No. CLNS-12-2084, 2013.

[3] J. Byrd, A. Chao, S. Heifets, M. Minty, T. O. Raubenheimer, J. Seeman, G. Stupakov, J. Thomson, and F. Zimmermann, Phys. Rev. Lett. 79, 79 (1997).

[4] J. Y. Huang, M. Kwon, T.-Y. Lee, I. S. Ko, Y. H. Chin, and H. Fukuma, Phys. Rev. Lett. 81, 4388 (1998).

[5] M. Kwon, J. Y. Huang, T.-Y. Lee, I. S. Ko, Y. H. Chin, H. Fukuma, M. Isawa, K. Ohmi, and M. Tobiyama, Phys. Rev. E 57, 6016 (1998).

[6] B. Jiang, G. Xia, L. Han, G. Liu, Z. Dai, and Z. Zhao, Nucl. Instrum. Methods Phys. Res., Sect. A 614, 331 (2010).

[7] M. Palmer et al., in Proceedings of the International Particle Accelerator Conference, Kyoto, Japan (ICR, Kyoto, 2010), p. 1191.
[8] J. Alexander et al., Nucl. Instrum. Methods Phys. Res., Sect. A 748, 96 (2014).

[9] A. Chatterjee, K. J. Blaser, M. P. Ehrlichman, D. L. Rubin, and J. P. Shanks, Fast Ion Instability at CESR-TA, in Proceedings of the International Particle Accelerator Conference 2014 (JACoW, Geneva, 2015), pp. 1638-1640.

[10] A. Oeftiger and G. Rumolo, CERN Report No. CERNOPEN-2011-050, 2011.

[11] W. Hager, Applied Numerical Linear Algebra (Prentice Hall, Englewood Cliffs, New Jersey, 1988).

[12] L. Wang, J. Safranek, Y. Cai, J. Corbett, R. O. Hettel, T. O. Raubenheimer, J. Schmerge, and J. Sebek, Phys. Rev. ST Accel. Beams 16, 104402 (2013).

[13] E. Kim and K. Ohmi, Jpn. J. Appl. Phys. 48, 086501 (2009). 